\title{
Bi-Criteria Smoothing of Data by Savitzky-Golay Filter
}

\author{
Venko G. Vitliemov ${ }^{1}$, Ivelin V. Ivanov ${ }^{1}$, Ivan A. Loukanov ${ }^{2}$ \\ ${ }^{1}$ Department of Technical Mechanics, Ruse University "Angel Kanchev”, Ruse, Bulgaria, \\ ${ }^{2}$ Department of Mechanical Engineering, University of Botswana, Gaborone, Botswana
}

\begin{abstract}
An approach for interactive bi-criteria smoothing of experimental data is proposed using the system $(S-G)$ filter of Salvitzky-Golay. An optimization problem is formulated with the controversial criteria: "total absolute error" and "integral smoothness". For the choice of adjusting parameters of the filter - the degree of the approximation of polynomial and the number of the supporting points is used. A Pareto-optimal solution set is determined for this problem. Then subsets of Pareto-optimal solutions are found by employing the $\boldsymbol{\mu}$-selection method, which are aranged by their extended efficiency. The solution with the highest rank of efficiency is the Salukvadze-optimum of the formulated extremal problem. The proposed approach allows choosing reasonably a variant of $(S-G)$ filter parameters having adequate representation and smoothness. An example of smoothing the measured values of an acceleration of free damped vibrations in the propulsion mechanism of a vibrobot is provided and analyzed.
\end{abstract}

Keywords: bi-criteria optimization, data smoothing, Savitzky-Golay filter, $\boldsymbol{\mu}$-selection, Salukvadze-optimum

\section{Introduction}

Very often the data of measured values of the magnitude of a particular variable carry additional noise of unknown frequency and source of origin that could compromise the data specified by the characteristics of the actual process. For example the parametric identification of one-dimensional damped oscillations of a queasylinear object accompanied by a high-frequency disturbance is hampered by an inaccurate determination of amplitude values, the queasy-period and the logarithmic decrement of decay of the oscillating process.

In such cases the analysis of the experimental data and the synthesis of an adequate theoretical model for their simulation generally require a prior smoothing of the data. For this reason the already developed methods and procedures for processing of signals with the help of smoothing filters are used. Filters are created with different complexity, accuracy, degree of smoothness, adaptability and efficiency [1], [2] [3] [4] [5] [6] [7]. The existing diversity of filters is conditioned by the above mentioned contradicting characteristics and filters inability to be combined together in a universal filter.

The development of more sophisticated filters cannot solve uniquely the main dilemma in any filtering the achievement of sufficient smoothness by removing much of the noise and the acceptable loss ratio to the data of the physical experiment [8]. The choice of unique compromise to the conflicting criteria is always a subjective act.

In this work interactive approach is proposed for solving this problem by selecting valid values of the controlled parameters of the subject filter, which are arranged in a compromised efficiency of Pareto-optimal solutions.

\section{Filter of Savitzki-Golay}

In the work [9] a classic version of a smoothing filter based on a method proposed by [10] is considered. The idea of the method is illustrated in Fig. 1. The smoothed value $a_{i}^{g}$ by the method of least squares (MLS) at the point $t_{i}$ is obtained as the value at that point of polynomial $p_{i}(t) \equiv p\left(t_{i}\right)$ of $n$ degree, approximating a set of creeping fixed number of adjacent measured points, $a_{i}^{e}, i=1,2, \ldots, n_{e}$. In Fig. 1 with $n_{L}$ the number of points, located to the left of the point $t_{i}$, and with $n_{R}$ - the number of points located on the right of the same point. The polynomial $p_{i}(t)$ uses as supporting $m=n_{L}+n_{R}+1$ points. In the process of smoothing the strip that contains $m$ points is moved incrementally to the right. At every new step it includes a new point on the right and the left endpoint is excluded.

Effective methods and computer programs for determining the coefficients $b_{k}$ of the approximation polynomial $p_{i}(t)=\sum_{k=0}^{n}\left[b_{k}\left(t-t_{i}\right) /\left(t_{i+1}-t_{i}\right)\right]^{k}$ are described in [2] and [3].

For the realization of the smoothing process with the (S-G)-filter, in this study the function sgolayfilt of the Signal Processing Toolbox of MATLAB system is used [11]. 


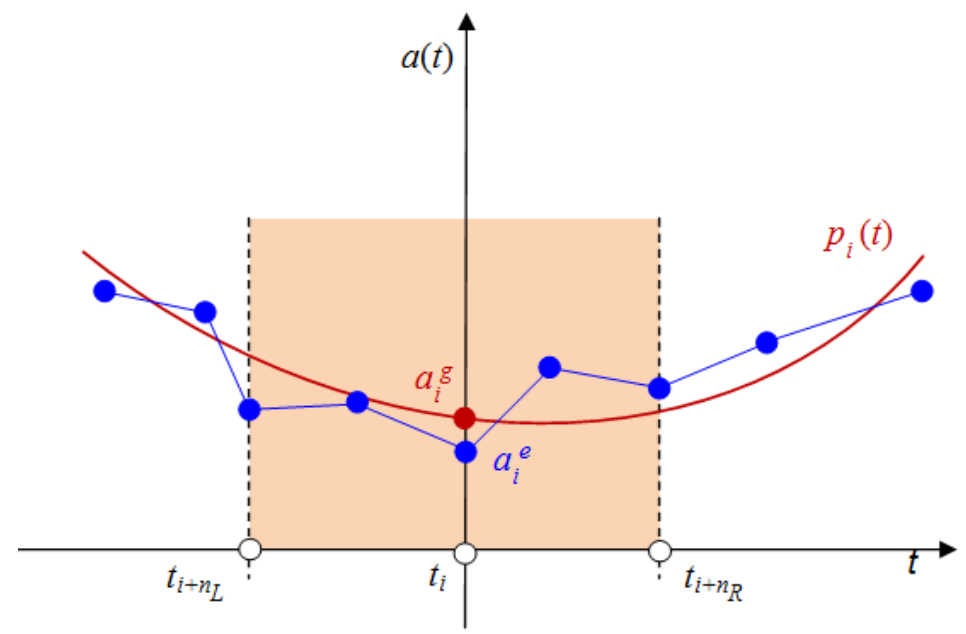

Fig. 1 shows smoothing with the polynomial $p_{i}(t)$ which is determined by the MLS

\section{Criteria for Optimality}

The choice of tunable parameters of the filter (S-G)-degree $n$ of the approximating polynomial and number $m$ of the supporting points in the smoothing strip is appropriate to be carried out by Pareto-optimal [12] values determined for the controversial criteria "total absolute error":

(1) $\quad J=\sum_{i=1}^{n_{e}}\left|a_{i}^{e}-a_{i}^{g}\right|$,

and the integral smoothness:

$$
I=\int_{0}^{t_{f}}\left[\mathrm{~d}^{2} a^{g}(t) / \mathrm{d} t^{2}\right]^{2} \mathrm{~d} t,
$$

where: $a_{i}^{e} \equiv a^{e}\left(t_{i}\right)$ and $a_{i}^{g} \equiv a^{g}\left(t_{i}\right)$ are the measured and smoothed values of the function $a(t)$ respectively and $n_{e}$ is the number of discrete values $a_{i}^{e}$ for $t_{i} \in\left[0, t_{f}\right], i=1,2, \ldots, n_{e}$.

The criterion (1) is a measure of adequacy of the smoothed values $a_{i}^{g}$ with respect to the measured $a_{i}^{e}$ ones. The criterion (2) characterizes the degree of smoothing of the approximating function $a_{i}^{g}$.

Since $a_{i}^{g}$ is a discrete function, the integral (2) is replaced with the nearest constant by the differential analog $I \approx \sum_{i=2}^{n_{e}-1}\left(a_{i+1}^{g}-2 a_{i}^{g}+a_{i-1}^{g}\right)^{2}$.

For determining the second derivative $\left.\left[a^{g}\left(t_{i}\right)\right)^{\prime \prime}\right]^{2}$ the MATLAB function "diff" is employed [13].

\section{Optimization Task}

The choice of optimal in terms of criteria (1) and (2) compromise values of the tunable parameters $n$ and $m$ of the (G-S)-filter can take place after solving the Bi-criteria extreme task (3):

$$
\begin{aligned}
& \mathbf{u}^{*}=\arg \operatorname{Pmin}_{\mathbf{u} \in \mathbf{D}} \mathbf{f}(\mathbf{u}), \\
& \mathbf{f}=[J(\mathbf{u}), I(\mathbf{u})], \mathbf{u}=[n, m], \\
& \mathbf{D} \equiv\left\{\mathbf{u} \in \mathbf{E}^{2}: \mathbf{u}^{-} \leq \mathbf{u} \leq \mathbf{u}^{+}\right\},
\end{aligned}
$$

where "Pmin" is the operator for determining the global Pareto-minimum [12] compromise values of the vector criterion $\mathbf{f}$ in fulfillment of the condition for fitting to the two-dimensional vector $\mathbf{u}$ of permitted multiple $\mathbf{D}$ being a rectangular area defined by given limiting values $-\mathbf{u}$ and $+\mathbf{u}$ of the vector $\mathbf{u}$.

\section{Optimization Procedure}

To solve the task (3) a modified version of the program "psims" for multi-criteria parametric optimization is used, as documented in [14]. Optimization is carried out in two stages [9].

In Stage 1 incremental calculation of the criterion vector $\mathbf{f}(\mathbf{u})$ for all points of the given net in the permissible area $\mathbf{D}$ is performed. For those points Pareto-optimal discrete sets $\mathbf{D}^{*}$ and $\mathbf{P}^{*}$ are determined approximately, in the parametric area $\mathbf{D}$ and in the reachable criteria region $\mathbf{P}$ respectively.

In Stage 2 arranged by a compromised efficiency Pareto-subsets are selected by using the minimum values $\mu_{k}^{*}, k \in \mathbf{I}_{\mathbf{k}} \equiv\{1,2,3\}$ of the components of the vector criterion $\boldsymbol{\mu}=\left[\mu_{1}, \mu_{2}, \mu_{3}\right]$ from the multiple $\mathbf{M} \equiv$ 
$\left\{\boldsymbol{\mu}\left(\mathbf{f}^{*}\right) \in \mathbf{E}^{3}: \mathbf{f}^{*} \in \mathbf{P}^{*}\right\}$. They correspond to the distances between three characteristic points: positive utopian point $\mathbf{f}^{\mathbf{U}}$ with components of uncompromised minima of particular criteria; the current compromise point $\mathbf{f}^{*}$ and its projection $\mathbf{f}^{\mathbf{U N}}$ on the strait line $U N$, which joins both utopian points - the positive $\mathbf{f}^{\mathbf{U}}$ and the negative $\mathbf{f}^{\mathbf{N}}$ with components uncompromised maximums of the particular criteria. In General, the minimum distances $\mu_{k}^{*}$ correspond to different Pareto-optimal point's $\mathbf{f}^{*}$.

With the help of vector criterion $\mu^{s}=\left[\mu_{1}{ }^{s}, \mu_{2}{ }^{s}, \mu_{3}{ }^{s}\right], s \in \mathbf{I}_{s} \equiv\left\{1,2, \ldots, N_{P}\right\}$, for each Pareto optimal point $\mathbf{f}^{* s} \in \mathbf{P}^{*}$ from the set $\mathbf{P}^{*}$ is transformed into a point of the multiple $\mathbf{M} \equiv\left\{\boldsymbol{\mu}^{s} \in \mathbf{E}^{3}: s \in \mathbf{I}_{\mathbf{s}}\right\}$ of the three dimensional $\boldsymbol{\mu}$-space. In $\mathbf{M}$ all possible combinations of two criteria $\left\{\mu_{t}^{s}, \mu_{h}^{s}\right\}, t \neq h, t, h \in \mathbf{I}_{\mathbf{k}}$ are investigated. For each pair of criteria subsets of Pareto-optimal points $\mathbf{M}_{\rho} \equiv\left\{\mu^{s} \in \mathbf{E}^{3}: \quad \mu_{t}{ }^{s, t} \leq \mu_{t}^{s} \leq \mu_{t}^{* s, h}, \quad h \neq t, \quad t, h \in \mathbf{I}_{\mathbf{k}}, \quad s \in \mathbf{I}_{\mathbf{s}}\right\} \subset \mathbf{M}$, $\rho \in \mathbf{I}_{\rho} \equiv\{1,2, \ldots, 6\}$ are selected, where the minimization point $\mu_{h}{ }^{s, h}=\min _{s \in \mathbf{I s}}\left\{\mu_{h}^{s}\right\}$ of a particular criterion $\mu_{h}^{s}$, $h \in \mathbf{I}_{\mathbf{k}}$ is used as an upper limit in the selection by another criterion $\mu_{t}^{s}, t \neq h, t \in \mathbf{I}_{\mathbf{k}}$, and the minimum point $\mu_{t}{ }^{s, t}=\min _{s \in \mathbf{I s}}\left\{\mu_{t}^{s}\right\}$ of the criterion $\mu_{t}^{s}-$ as a lower limit. Every selected point that way $\boldsymbol{\mu}^{\circ} \in \mathbf{M}_{\rho}, \rho \in \mathbf{I}_{\rho}$, and therefore its corresponding point $\mathbf{f}^{\mathbf{0}} \in \mathbf{P}_{\boldsymbol{R}} * \subset \mathbf{P} *$ receive as an individual assessment a number $R_{E}=\max \{\rho\}$, which specifies its rank of a compromising efficiency. This figure corresponds to the number $\rho$ of the subsets $\mathbf{M}_{\rho}$ in the combined multiple $\mathbf{M}_{R} \equiv\left\{\cap_{\rho \in \mathbf{I} \rho} \mathbf{M}_{\rho}\right\}$, to which $\boldsymbol{\mu}^{\circ} \equiv \boldsymbol{\mu}\left(\mathbf{f}^{\circ}\right)$ belongs. The subset of $\mathbf{P}_{\boldsymbol{R}}{ }^{*}$ with the highest rank $R_{E}=6$ usually contains only one point $\mathbf{f}^{\mathbf{S}} \equiv \mathbf{f}\left(\mathbf{u}^{\mathbf{S}}\right)$, which corresponds to the Salukvadze- optimal solution $\left(\mathbf{u}^{\mathbf{S}} \equiv \equiv \arg \min _{\mathbf{u} \in \mathbf{D}} \mu_{3}(\mathbf{f}(\mathbf{u})), \mathbf{f}^{\mathbf{s}} \equiv \mathbf{f}\left(\mathbf{u}^{\mathbf{S}}\right)\right)$ of task (3), as suggested in [15]. This decision reveals the potentials for an even approach of the particular criteria to their uncompromised optimal values under the assumption that they are equivalent.

The final compromise solution $\left(\mathbf{u}^{\#}, \mathbf{f}^{\#}\right)$ can be chosen after analysis of the ranged Pareto-optimal subsets in $\mathbf{P}_{\boldsymbol{R}} *$. First the Salukvadze optimal solution is analyzed. If it is assessed as unacceptable on the reached level of compromise by any of the particular criteria, then the subsets of $\mathbf{P}_{R}{ }^{*}$ with a lower rank are consistently analyzed until a definite choice is made.

\section{Example}

The proposed optimization approach will be applied for the smoothing of the measured acceleration of free damped oscillations of the propulsion mechanism of a vibrobot studied in [16] and experimentally investigated in [8]. The mechanical model of the tested vibrobot is shown schematically in Fig. 2.

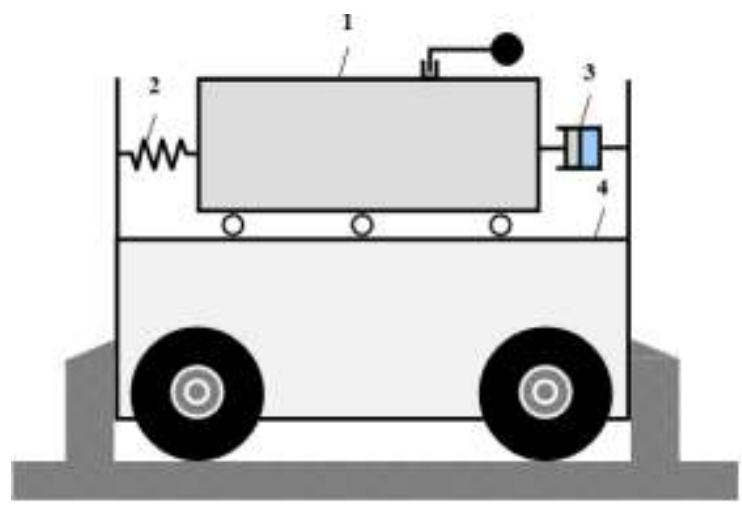

Fig. 2 displays the mechanical model of the vibrobot, where: 1 - is the propulsion mechanism; 2- equivalent spring; 3 - equivalent damper; 4 - temporary fixed chassis just for the experiments

Fig. 3 shows the diagram of the measured acceleration $a_{i}^{e} \equiv a^{e}\left(t_{i}\right)$ at discrete instants of time $t_{i}$ depicted with constant step $0.001 \mathrm{~s}$ after deviating and releasing the propulsion mechanism from its equilibrium position. It may be seen that the measured data contain a high-frequency disturbance, which fades gradually together with the main oscillating process [8].

Fig. 4 presents a fragment of the amended absolute values $\left|a_{i}^{e}\right|$, which illustrates a close-up of the high frequency disturbance observed in the acceleration record.

For compromise smoothing of the acceleration $a_{i}^{e}$ with the (G-S)-filter the task (3) is solved in area D with boundary values $\mathbf{u}^{-}=[2,31], \mathbf{u}^{+}=[10,71]$ of the parametric vector $\mathbf{u}$. In the area $\mathbf{D}$ a net of 189 trial points is built of which 33 are Pareto-optimal.

The established utopian points $\mathbf{f}^{\mathbf{U}}=[202.4,0.2815], \mathbf{f}^{\mathbf{N}}=[288.7,6.173]$ specify a straight line $U N$ located into the criteria space that defines the direction of the coordinated improvement of the values of the partial criteria (in the direction of $N$ to $U$ ). The components of the ideal point $\mathbf{f}^{\mathbf{U}}$ are the uncompromised minimums $J^{*}$ and $I^{*}$ of the introduced partial criteria (1) and (2). 


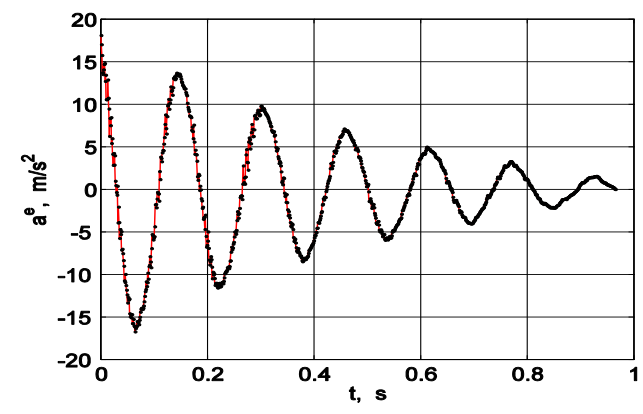

Fig. 3 shows the measured values $(\cdot)$ of the acceleration $\boldsymbol{a}^{e}(\boldsymbol{t})$

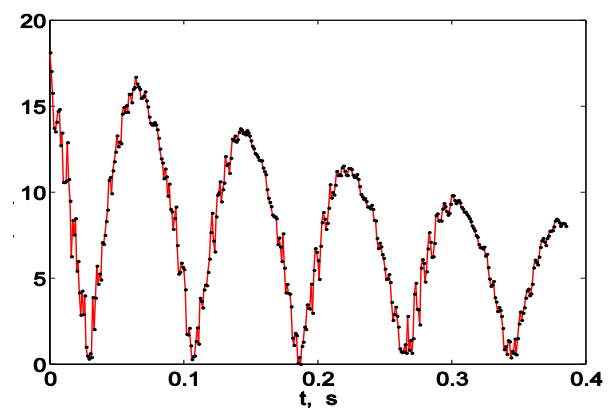

Fig. 4 illustrates absolute values $\left|\boldsymbol{a}^{\boldsymbol{e}}\left(\boldsymbol{t}_{\boldsymbol{i}}\right)\right|$ of the measured acceleration $a^{e}(t)$ за $t$

With the help of $\mu$-selection technique from the Pareto-optimal multitude five un-empty subsets with rank $R_{E} \in\{6,5,4,2,1\}$ are found. The results of this selection are presented partially in Table 1 . The compromised solution $S$ with the highest rank $R_{E}=6$ is the Salukvadze-optimum of the task (3).

Table1. Ranged Pareto-Optimal Solutions

\begin{tabular}{|l|l|l|l|l|l|}
\hline Solution & \multirow{2}{*}{$R_{E}$} & \multicolumn{3}{|c|}{$\mathbf{u}$} & \multicolumn{1}{c|}{} \\
\cline { 3 - 6 } & & $n$ & $m$ & $J, \mathrm{~m} / \mathrm{s}^{2}$ & $I, \mathrm{~m}^{2} / \mathrm{s}^{4}$ \\
\hline S & 6 & 8 & 57 & 229.4 & 1.411 \\
\hline A & 5 & 6 & 41 & 229.0 & 1.867 \\
\hline & 5 & 8 & 71 & 236.1 & 0.8768 \\
\hline & 4 & 7 & 51 & 231.5 & 1.327 \\
\hline & 4 & 7 & 71 & 238.4 & 0.5843 \\
\hline & 2 & 10 & 51 & 225.3 & 2.474 \\
\hline & 2 & 5 & 59 & 241.3 & 0.5009 \\
\hline B & 1 & 10 & 49 & 221.1 & 2.739 \\
\hline C & 1 & 5 & 71 & 246.7 & 0.3988 \\
\hline
\end{tabular}

Fig. 5 illustrates the achieved smoothness and closeness of the Salukvadze-optimal solution in comparison to the measured acceleration.

The Fig. 6 shows all test points in the field $\mathbf{D}$ after realization of Stage 2 of the optimization procedure. In this and the following figures the point with the highest rank $R_{E}=6$ is marked with the symbol ( $\square$ ) and the points of rank $5,4,2,1-$ with $(\bullet, \boldsymbol{\Delta}, 4, \bullet)$, respectively.

Fig. 7 gives visual appreciation for the location of the Pareto-optimal points in the $\mu$-space relative to the utopian points $\boldsymbol{\mu}^{\mathrm{U}}$ and $\boldsymbol{\mu}^{\mathrm{N}}$ in this space, marked by the symbol $(\bullet)$ and the straight line which connects them. This figure allows to make a visual assessment of the nearness (proximity) of the ranged points to the utopian point $\boldsymbol{\mu}^{\mathbf{U}}$ and to identify alternative options of solutions in the criteria space.

In Fig. 8 the normalized by the scheme $J^{\mathrm{o}}=J /\left(J^{\mathrm{N}}-J^{\mathrm{U}}\right), I^{\mathrm{o}}=I /\left(I^{\mathrm{N}}-I^{\mathrm{U}}\right)$ Pareto-front and the ranged points of the conducted $\mu$-selection is depicted.

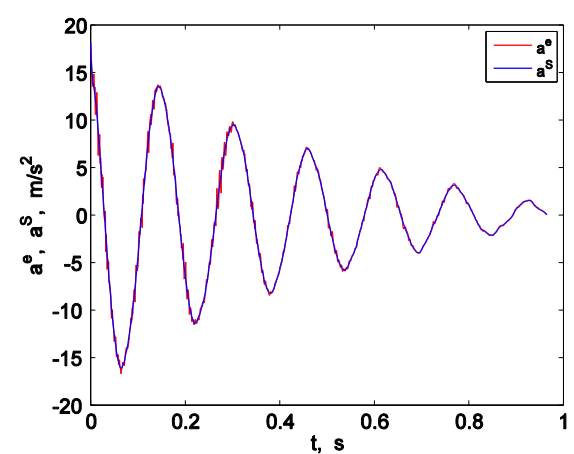

Fig. 5 illustrates the measured $\boldsymbol{a}^{\boldsymbol{e}}$ and smoothed Salukvadze optimum acceleration $\boldsymbol{a}^{\mathbf{S}}$.

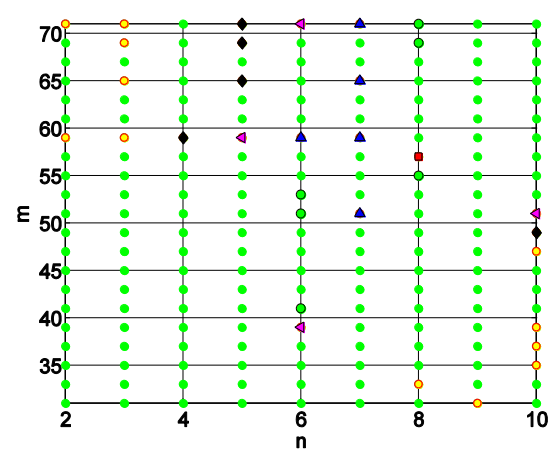

Fig. 6 illustrates the permissible $(\bullet)$, Pareto-optimal (०) and the ranked points in area $\mathbf{D}$

From Fig. 9 we find out that the solutions $a^{\mathrm{S}}$ and $a^{\mathrm{A}}$ are explicitly hard to distinguish from each other. This could be the basis of an alternative to the solution $S$ for the final compromise solution $\left(\mathbf{u}^{\#}, \mathbf{f}^{\#}\right)$ of the task (3) to prefer selecting the option A. 


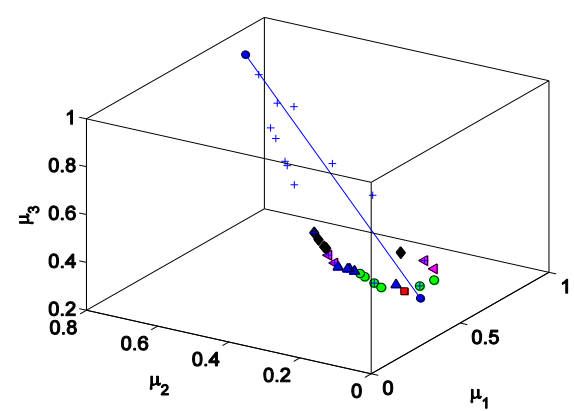

Fig. 7 illustrates Pareto-optimal $(+)$ and ranged points in the criteria $\boldsymbol{\mu}$-space

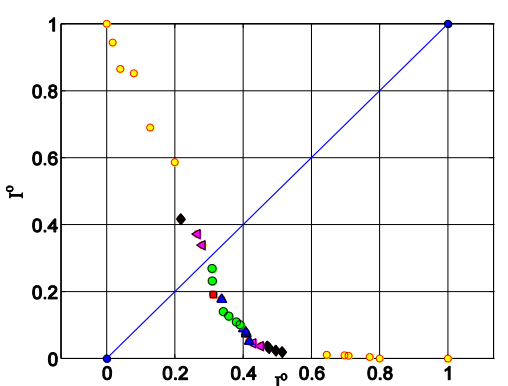

Fig. 8 shows normalized Paretooptimal (•) and ranged criteria

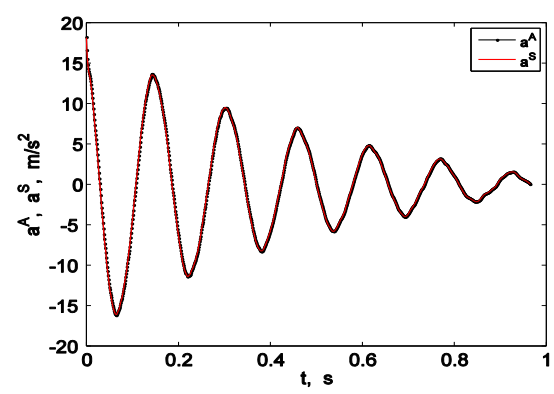

Fig. 9 illustrates the smoothed Paretooptimal accelerations $\boldsymbol{a}^{\mathbf{S}}$ and $\boldsymbol{a}^{\mathbf{A}}$

\section{Conclusions}

The proposed approach for interactive bi-criteria smoothing of experimental data with the system filter (S-G) allows practically approximating experimental data with selected smoothness and adequacy. This method may be used in using experimental data for any theoretical analysis and also for practical purposes when the period, logarithmic decrement and amplitudes need to be determined from experiments with high precision.

There are many practical applications of the proposed approach when high accuracy is required, for example, whenever resonance frequency has to be determined, like for sonic inertia-powered pumps, vibrationdriven robots, hammer drills, vibration screens and many other machineries operating in resonance.

\section{References}

[1] Browne, M., N. Mayer, T.R.H. Cutmore. A multiscale polynomial filter for adaptive smoothing (Digital Signal Processing, Vol. 17, $69-75,2007)$

[2] Gander, W., J. Hřebíček. Solving Problems in Scientific Computing Using Maple and MATLAB (Lerlin, Springer, Berlin, 2004).

[3] Hamming, R.W. Digital Filters (Prentice-Hall, Englewood Cliffs, NJ, 1989).

[4] Oppenheim, A.V., R. W. Schafer. Discrete-Time Signal Processing (Pearson, Upper Saddle River, NJ, 2010).

[5] Orfanidis, S.J. Introduction to Signal Processing (Prentice Hall, Upper Saddle River, 2009) http://www.ece.rutgers.edu/ orfanidi/intro2sp .

[6] Persson, P.-O., G. Strang. Smoothing by Savitzky-Golay and Legendre filters. J. Rosenthal et al. (Eds.), Mathematical Systems Theory in Biology, Communications, Computations, and Finance (Springer, New York, 301-316, 2003)

[7] Wrobel, I., Zietak, K. On the Legendre-based filters of Persson and Strang. Applied Mathematics and Computation. Vol. 218, No. 8, 4 216-4233, 2011.

[8] Loukanov I.A., S.P. Stoyanov Experimental determination of dynamic characteristics of a vibration-driven robot. IOSR Journal of Mechanical and Civil Engineering, Vol. 12, No. 3, 62-73, 2015b

[9] Cheshankov, B.I., I.V. Ivanov, V.G. Vitliemov, P.A. Koev. PSI-method Multi-criteria Optimization Contracting the Set of Tradeoff Solutions. 15th International Conference on Systems Science, Wroclaw, Poland, Vol. 1, 281-288, 2004.

[10] Savitzky, A., M.J.E. Golay. Smoothing and differentiation of data by simplified least-squares procedures. Analytical Chemistry, Vol. 36, No. 8, 1627-1639, 1964.

[11] Signal Processing Toolbox ${ }^{\mathrm{TM}}$ User's Guide. (MathWorks, Inc., Natick, MA, 2015), http://www.mathworks.com/help/pdf_doc/signal/signal tb.pdf

[12] Ehrgott, M. Multicriteria Optimization (Springer, Berlin, 2005).

[13] Symbolic Math Toolbox TM User's Guide. (MathWorks, Inc., Natick, MA, 2015) https://www.mathworks.com/help/pdf_doc/symbolic/symbolic_tb.pdf

[14] Йорданов, Й.Т., В.Г. Витлиемов. Оптимизация с МATLAB. Прагматичен подход. Университетско издателство „Ангел Кънчев“", Русе, 2013, http://ecet.ecs.uni-ruse.bg/else/subjects/_index.php?cid=2130211020910970.

[15] Salukvadze, M.E. Vector-Valued Optimization Problems in Optimal Control Theory (Academic Press, New York, 1979)

[16] Loukanov, I.A. Inertial Propulsion of a Mobile Robot. IOSR Journal of Mechanical \& Civil Engineering, Vol. 12, No. 2, 23-33, $2015 \mathrm{a}$. 